\title{
Gabriele Jancke
}

\section{Patronagebeziehungen in autobiographischen Schriften des 16. Jahrhunderts - Individualisierungsweisen?*}

Gelehrte gelten (neben Künstlern) als die klassische soziale Gruppe, mit der die Individualisierung beginnt. Gleichzeitig sind Gelehrte aber auch genau die Gruppe, die in ihren Selbstzeugnissen ihre Patronagebeziehungen mitsamt ihren Abhängigkeiten offenlegt. Individualisierung und Patronage eröffnen also ein Spannungsfeld, das, wie mir scheint, nicht mit einfachen Antworten schnell wieder zu schließen ist. Das Spannende an diesem Feld sehe ich nicht unbedingt darin, daß man sich nun entscheiden müßte zwischen Individualisierung und Patronage, nach dem Modell eines Entweder-Oder; auch nicht darin, daß nun abzuwägen wäre, wie viel Individualisierung und wie viel Patronage jeweils vorhanden gewesen wären. In diesem Fall bestünde das Spannungsfeld nur aus einem Gegenüber von zwei unverbundenen Polen und wäre damit nach dem Muster bipolarer, gegensätzlicher Kategorien organisiert ${ }^{1}$.

Warum aber sollte das wirklich so sein? Was läßt uns annehmen, daß Individualisierung und Patronagebeziehungen im Gegensatz zueinander stehen? Konnte Individualisierung nur ohne Patronagebeziehungen stattfinden? Waren Patronagebeziehungen so beschaffen, daß sie keine Individualisierung erlaubten? Wie wäre Individualisierung dann überhaupt möglich gewesen in den europäischen Gesellschaften, die doch vom 15. bis weit ins 19. Jahrhundert hinein tief von Patronageverhältnissen geprägt waren²?

* Bedanken möchte ich mich bei der DFG, die diese Arbeit im Rahmen der Forschergruppe "Selbstzeugnisse in transkultureller Perspektive" gefördert hat, und bei Claudia Ulbrich für höchst anregende Gespräche über Individualität und die vielen offenen Fragen in diesem Zusammenhang.

1 Kritisch zu solchen dichotomen Mustern, die mit dem Begriff des Individuums meist verbunden sind: Gabriele Jancke, Claudia Ulbrich, Vom Individuum zur Person. Neue Konzepte im Spannungsfeld von Autobiographietheorie und Selbstzeugnisforschung, in: Vom Individuum zur Person. Neue Konzepte im Spannungsfeld von Autobiographietheorie und Selbstzeugnisforschung, hrsg. v. Gabriele Jancke, Claudia Ulbrich (Querelles. Jahrbuch für Frauen- und Geschlechterforschung 10, Göttingen 2005) 1-27.

2 Dazu nur einige ausgewählte Titel, wobei ich mich bewußt auf die modernen westlichen Industriestaaten und deren politische Strukturen konzentriere: Huw Beynon, T. Austrin, Masters and Servants: Class and Patronage in the Making of a Labour Organisation (London 1990); J. M. Bourne, Patronage and Society in Nineteenth-Century England (London 1986); 


\section{Individualisierung - was ist das?}

In der Geschichtswissenschaft wird, wie Winfried Schulze in seinem Aufsatz „Das Wagnis der Individualisierung“ präzise zusammenfaßt, „unter Individualisierung ein Oppositum zur Einbindung des Menschen in umfassendere Sozialverbände gesehen“, und er fährt fort: „In diesem Zusammenhang bedeutet Individualisierung Befreiung von Bindungen, die dem jeweiligen Verband eigen sind, Abkehr von den jeweiligen Normensystemen, Nutzung neuer Ausdrucksformen." ${ }^{\text {3 }}$ Als Beispiele für solche einbindenden Sozialverbände nennt Schulze den Haushalt bzw. das Brunnersche Konzept des „ganzen Hauses“, die Kirche, den Geburtsstand, die Stadt, die Zunft. Nach Schulze sind es also nicht schon beliebige soziale Beziehungen oder Traditionen als solche, die einer Individualisierung entgegenstehen.

Während hier und in vielen anderen historischen Arbeiten Individualisierung meist als Emanzipation und als Weg in die persönliche Autonomie aufgefaßt wird, zeigt Flavia Kippeles Untersuchung, daß dies nur einen kleinen Ausschnitt der in der Soziologie diskutierten Merkmale darstellt ${ }^{4}$. Für die soziologischen Klassiker wie Marx und Engels, Tönnies, Simmel, Durkheim, Weber und Elias konnte Individualisierung zwar ebenfalls Autonomie oder die Emanzipation aus traditionellen Bindungen sein, außerdem aber auch Freiheit, persönliche Eigenart, eine positivere Bewertung des Individuums, innerpsychische Differenzierung, Bewußtwerdung des eigenen Ichs, ein Rückzug ins Private; auch so negativ empfundene Phänome-

Christopher Clapham, Clientelism and the State, in: Private Patronage and Public Power. Political Clientelism in the Modern State, hrsg. v. Christopher Clapham (London 1982) 1-35; Ursula Fubrich-Grubert, Der Oberpräsident Theodor von Schön. Ein Liberaler im Kampf um Preußens permanente Reform?, in: Preußens Weg in die politische Moderne. Verfassung - Verwaltung - politische Kultur zwischen Reform und Reformblockade, hrsg. v. Bärbel Holtz, Hartwin Spenkuch (Berichte und Abhandlungen, Berlin-Brandenburgische Akademie der Wissenschaften, Sonderband 7, Berlin 2001) 29-58; Lutz Raphael, Recht und Ordnung. Herrschaft durch Verwaltung im 19. Jahrhundert (Europäische Geschichte, Frankfurt a. M. 2000); Ronald M. Sunter, Patronage and Politics in Scotland, 1707-1832 (Edinburgh 1986); Wolfgang Weber, Priester der Klio. Historisch-sozialwissenschaftliche Studien zur Herkunft und Karriere deutscher Historiker und zur Geschichte der Geschichtswissenschaft 1800-1970 (EHS III 216, Frankfurt a. M. etc. ${ }^{21987, ~ 11984) ; ~ E r i c ~ W o l f, ~ K i n s h i p, ~}$ Friendship, and Patron-Client Relations in Complex Societies, in: The Social Anthropology of Complex Societies, hrsg. v. Michael Banton (New York 1966) 1-22, Nachdr. in: Friends, Followers and Factions: A Reader in Political Clientelism, hrsg. v. Steffen W. Schmidt, Laura Guasti, Carl H. Landé, James S. Scott (Berkeley 1977) 167-177.

3 Winfried Schulze, Das Wagnis der Individualisierung, in: Wege in die Neuzeit, hrsg. v. Thomas Cramer (Forschungen zur Geschichte der älteren deutschen Literatur 8, München 1988) 270-286, hier 272.

4 Flavia Kippele, Was heißt Individualisierung? Die Antworten soziologischer Klassiker (Opladen 1998), nützlich v. a. Kap. 9: Dimensionen der Individualisierung 200-242. Verschiedene Typen von Individualität unterscheidet Hans-Georg Soeffner, ohne dabei allerdings Bezüge zu den umgebenden Gesellschaftsstrukturen oder soziale Zuordnungen zu klären: Hans-Georg Soeffner, „Typus und Individualität“ oder „Typen der Individualität“? - Entdeckungsreisen in das Land, in dem man zuhause ist, in: Typus und Individualität im Mittelalter, hrsg. v. Horst Wenzel (Forschungen zur Geschichte der älteren deutschen Literatur 4, München 1983) 11-44, hier 30-41. 
ne wie Isolierung oder Ohnmacht werden genannt. Bemerkenswerterweise finden sich auch Kosmopolitanismus und soziale Vernetzung unter den möglichen Charakteristika von Individualisierungsprozessen. Mit der „Übernahme von Werten“ als mögliche Variante von Autonomie ist schließlich auch eine traditions- oder gruppenorientierte Haltung unter den Individualisierungsweisen vertreten. Folgt man also den Soziologen, dann wäre Individualisierung zumindest mit einem Teil ihrer möglichen Merkmale stark in die jeweilige Gesellschaft und in konkrete soziale Beziehungen, Orientierungen und Wertsysteme eingebettet.

In einer Fülle von historischen Arbeiten ist eine kaum übersehbare Vielfalt von Dimensionen mehr angedeutet als reflektiert worden, die mit Individualisierungsprozessen angesprochen sein könnten ${ }^{5}$. Was ist nun konkret unter Individualisierung zu verstehen? Es lohnt sich, die Fragen zusammenzutragen und genau zu stellen.

Was ist es, was man in einer Bewegung der Individualisierung erreicht? Bedeutet es, ein eigenes Zimmer zu haben? In einem eigenen Bett zu schlafen, oder auch: allein in einem Bett zu schlafen? Beim Essen einen festen Platz am Tisch zu haben, eine eigene Sitzgelegenheit, eigenes Geschirr, eigenes Besteck? Heißt es, daß sich die Mahlzeiten nach den eigenen Zeiten und Bedürfnissen richten? Bedeutet es, eigene und ungebrauchte Kleidung zu haben? Heißt es, daß man in seinem eigenen Haus wohnt? Daß man eigenen Grundbesitz hat? Daß man seinen eigenen Haushalt und seinen eigenen Lebensunterhalt hat? Heißt es, daß man sich frei bewegen kann? Oder eher, daß man einen festen Ort hat ${ }^{6}$ ?

Wen betrifft der Prozess der Individualisierung - alle Mitglieder einer Gesellschaft gleichermaßen? Nur oder bevorzugt Männer ${ }^{7}$ ? Vorwiegend die Eliten?

5 Auch Martin Scheutz und Harald Tersch machen auf die Unklarheit dessen aufmerksam, was jeweils mit „Individualisierung“ gemeint ist: „Der dynamische Begriff der ,Individualisierung' und des ,modernen Individuums' - was immer sich auch dahinter verbergen mag bleibt unscharf und ist problematisch.", Martin Scheutz, Harald Tersch, Individualisierungsprozesse in der Frühen Neuzeit? Anmerkungen zu einem Konzept, in: Wiener Zeitschrift zur Geschichte der Neuzeit 1,2 (2001) 38-59, hier 39; im folgenden zitiert: Scheutz, Tersch, Individualisierungsprozesse.

6 Für solche konkreten, aufs Materielle zielenden Fragen vgl. etwa: Geschichte des privaten Lebens, Bd.3: Von der Renaissance zur Aufklärung, hrsg. v. Philippe Ariès, Roger Chartier (Frankfurt a. M. 1991, zuerst frz. 1986); Raffaella Sarti, Europe at Home. Family and Material Culture, 1500-1800 (New Haven, London 2002, zuerst it.: Vita di casa. Abitare, mangiare, vestire nell'Europa moderna, 1999); Alan Macfarlane, The Origins of English Individualism. The Family, Property and Social Transition (Oxford 1978).

7 Die feministische Forschung hat schon länger nachdrücklich auf die Tatsache hingewiesen, daß Individualitätskonzepte sich einseitig, wenngleich meist implizit bleibend, auf Männer beziehen, vgl. etwa $R$. W. Connell, The Big Picture: Masculinities in Recent World History, in: Theory and Society 22 (1993) 597-623; Karin Hausen, Die Nicht-Einheit der Geschichte als historiographische Herausforderung. Zur historischen Relevanz und Anstößigkeit der Geschlechtergeschichte, in: Geschlechtergeschichte und Allgemeine Geschichte. Herausforderungen und Perspektiven. Mit Beiträgen v. Karin Hausen, Lynn Hunt, Thomas Kühne, Gianna Pomata und Helmut Puff, hrsg. v. Hans Medick, Anne-Charlott Trepp (Göttinger Gespräche zur Geschichtswissenschaft 5, Göttingen 1998) 15-55, hier 45; Lynn Hunt, The Challenge of Gender. Deconstruction of Categories and Reconstruction of Narratives in 
Mehr die städtische Bevölkerung oder auch die ländliche8? Welche Rolle spielen Kategorien wie Geschlecht, Stand, Religion, Alter?

Bedeutet Individualisierung, daß man selbst bestimmen kann, mit wem man ißt, schläft, arbeitet, sich aufhält, spricht, oder wann und wie man es tut? Heißt es, daß man seine Ehepartnerin oder frau ibren Ehepartner selbst wählen kann? Heißt es, daß man Zugang hat zu den Personen und Gruppen, bei denen man es möchte, also daß man Beziehungen knüpfen und Bindungen aufbauen kann? Oder bedeutet es, daß man Bindungen abbrechen, Beziehungen verlassen, Zugehörigkeiten beenden kann? Heißt es, daß man dann ohne Bindungen und Zugehörigkeiten sein kann oder sein $m u ß$ ? Bedeutet es, allein zu sein, isoliert, ohne Unterstützung und hilfreiche Beziehungen? Oder heißt es, allein sein zu können, wenn man allein sein will - aber dies vor dem Hintergrund von funktionierenden Beziehungen? Bedeutet es, daß man von anderen wahrgenommen, gewürdigt und respektiert wird? Heißt es, daß man öffentlich auftritt? Daß man seine Ansichten sagen oder schreiben kann, und daß diese auch von anderen gefragt sind? Bedeutet es, Einfluß auf andere zu haben oder Autorität? Daß man in seinem Haushalt etwas zu sagen hat? $\mathrm{Da}$ man bei politischen Entscheidungen etwas mitzureden hat? Heißt es, daß man Entscheidungen treffen kann und auch für verantwortlich gehalten wird ${ }^{9}$ ?

Heißt Individualisierung, daß man über sich reflektiert, daß man ein Bewußtsein von sich hat, daß man sich als anders empfindet als andere, daß man sich als etwas Besonderes einschätzt, daß man etwas über sich herausfinden will, daß man liest, daß man für sich allein liest, daß man überhaupt schreiben kann, daß man viel schreibt, daß man eine persönliche Handschrift entwickelt, daß man sich im Bild

Gender History, in: ebd. 57-97, hier 72; Andrea Maibofer, Geschlecht als Existenzweise. Macht, Moral, Recht und Geschlechterdifferenz (Aktuelle Frauenforschung, Frankfurt a. M. 1995) 36f., 150-155; Andrea Günter, Vom Wunsch, ein Stück Schokolade zu essen. Oder: Was bedeuten Autonomie und Freiheit in der Krankenpflege?, in: dies., Weibliche Autorität, Freiheit und Geschlechterdifferenz. Bausteine einer feministischen politischen Theorie $(\mathrm{Fa}-$ cetten, Königstein i.Ts. 1996) 230-242, hier 232-235; Henrietta L. Moore, Mensch und Frau sein. Perspektiven einer feministischen Anthropologie. Mit einer Einf. v. Herlinde PissarekHudelist (Gütersloh 1990, zuerst engl. 1988) 78-83: Der Traum von der autonomen Persönlichkeit; Ursula I. Meyer, Einführung in die feministische Philosophie (München 1997, ND Aachen 21994; zuerst: ebd. ${ }^{1}$ 1992), s. ebd. Register unter „Individualität“ und „Individuum“; Seyla Benhabib, Der verallgemeinerte und der konkrete Andere. Ansätze zu einer feministischen Moraltheorie, in: Denkverhältnisse. Feminismus und Kritik, hrsg. v. Elisabeth List, Herlinde Studer (Frankfurt a. M. 1989) 454-487.

8 Vgl. Jan Peters, Wegweiser zum Innenleben? Möglichkeiten und Grenzen der Untersuchung popularer Selbstzeugnisse der Frühen Neuzeit, in: Historische Anthropologie 1 (1993) 235-249, bes. 246-249, der auf Phänomene von Individualität in der ländlichen Gesellschaft hinweist; ferner Werner Troßbach, Individuum und Gemeinde in der ländlichen Welt, in: Entdeckung des Ich. Die Geschichte der Individualisierung vom Mittelalter bis zur Gegenwart, hrsg. v. Richard van Dülmen (Köln, Weimar, Wien 2001) 197-217.

$9 \mathrm{Zu}$ politischen und Öffentlichkeitsdimensionen vgl. etwa: The Individual in Political Theory and Practice, hrsg. v. Janet Coleman (The Origins of the Modern State in Europe, 13th to 18th Centuries, Oxford, New York 1996); C. B. Macpherson, Die politische Theorie des Besitzindividualismus. Von Hobbes bis Locke (Frankfurt a. M. 1967, zuerst engl.: The Political Theory of Possessive Individualism. Hobbes to Locke [Oxford 1962]). 
porträtiert, daß man eine Autobiographie oder ein Tagebuch schreibt, daß man eine Autobiographie veröffentlicht, daß man Schriftsteller oder Künstler ist ${ }^{10}$ ?

Auf welchen Ebenen von Geschichte ist Individualisierung anzusiedeln? Handelt es sich um etwas, was sich im individuellen Leben von einzelnen Menschen abspielt? Oder handelt es sich um die Werte und Strukturen einer Gesellschaft, ein formelles oder informelles Ethos, das bestimmte Verhaltensweisen fordert, fördert, privilegiert und $\mathrm{zu}$ einem grundlegenden Muster des Selbstverständnisses als Gesellschaft erhebt? Oder schließlich: Handelt es sich um ein den historischen Akteuren und Akteurinnen mehr oder weniger bewußtes Muster eines übergreifenden, langfristigen historischen Prozesses, in dessen Verlauf Individualisierung entsteht, sich ausbreitet und schließlich zu einem markanten, entscheidenden und unterscheidenden Merkmal bestimmter Gesellschaften im globalen Maßstab wird ${ }^{11}$ ? Wird Individualität positiv bewertet oder als Mangel gesehen ${ }^{12}$ ? Wer überhaupt sind die Akteurinnen und Akteure von Individualisierung? Sind die Menschen, die Individualisierung am eigenen Leibe und an der eigenen Person erfabren, immer zugleich die Akteure und Akteurinnen? Findet Individualisierung freiwillig statt? Wird sie bewußt und absichtlich angestrebt? Oder ergibt sie sich unabsichtlich oder auch wider Willen, weil andere Optionen nicht zur Verfügung stehen oder als schlechter eingeschätzt werden? Können Individualisierungsweisen unter Umständen auch Marginalisierungsweisen sein? Was passiert, wenn bestimmte Individualisierungsweisen $\mathrm{zu}$ einem allgemeinen Phänomen werden? Sollten wir dann auch gleichzeitig von Normierungs- und Homogenisierungsweisen sprechen?

Und schließlich: Woran erkennen wir Individualisierung? Wie entscheiden wir, ob Individualisierung real im Leben von einzelnen Menschen stattfand, real in der ganzen Gesellschaft eine Rolle spielte? Welche Zeichen lesen wir als Indizien?

10 Zur engen Verknüpfung von Individualität und autobiographischem Schreiben vgl. etwa Martin A. Danahay, Art. Individualism and Life Writing. In: Encyclopedia of Life Writing. Autobiographical and Biographical Forms, hrsg. v. Margaretta Jolly, 2 vols. (London, Chicago 2001) 466f., hier 466; Richard van Dülmen, Die Entdeckung des Individuums, 15001800 (Europäische Geschichte, Frankfurt a. M. 1997); Entdeckung des Ich. Die Geschichte der Individualisierung vom Mittelalter bis zur Gegenwart, hrsg. v. Richard van Dülmen (Köln, Weimar, Wien 2001), sowie kritisch dazu die Rezension von Gabriele Jancke, in: L'Homme Z.F.G. 13,2 (2002) 297-300, kritisch ferner Scheutz, Tersch, Individualisierungsprozesse 44f.: „Van Dülmen übernimmt nicht nur den Autorenkanon Mischs, sondern auch dessen Urteile über die Einzelwerke... Damit wird gleichzeitig einem bestimmten Individualitätsmodell seine Reverenz erwiesen." (45).

${ }^{11} \mathrm{Zu}$ dieser eminent wichtigen methodischen Unterscheidung s. Dieter Kartschoke, IchDarstellung in der volkssprachigen Literatur, in: Entdeckung des Ich. Die Geschichte der Individualisierung vom Mittelalter bis zur Gegenwart, hrsg. v. Richard van Dülmen (Köln, Weimar, Wien 2001) 61-78, v. a. 77f.; im folgenden zitiert: Kartschoke, Ich-Darstellung.

12 Vgl. Kartschoke, Ich-Darstellung 78. - Zu mittelalterlichen Porträts vgl. den wichtigen Aufsatz von Bruno Reudenbach, Individuum ohne Bildnis? Zum Problem künstlerischer Ausdrucksformen von Individualität im Mittelalter, in: Individuum und Individualität im Mittelalter, hrsg. v. Jan A. Aertsen, Andreas Speer (Miscellanea Mediaevalia 24, Berlin 1996) 807-818. Den Hinweis auf diesen Aufsatz verdanke ich Sigrid Ruby. 
Können wir für alle Kontexte mit den gleichen Indizien rechnen? Wie überprüfen wir, ob bestimmte Sachverhalte oder Quellensorten den Stellenwert hatten, den wir ihnen zumessen ${ }^{13}$ ? Welche Rolle haben Selbstzeugnisse oder Selbstporträts gespielt? Wie stellen wir fest, ob einzelne Elemente nicht nur isolierte Teile im Rahmen von anders ausgerichteten Verhältnissen waren, sondern sich addierten zu einer sozialen Struktur, einem gesellschaftlichen Selbstverständnis oder sogar zu einem zusammenhängenden, langfristigen Prozess der Individualisierung ${ }^{14}$ ? Welche Rolle spielt der kulturelle Kontext, um Phänomene in die Perspektive von Individualisierung zu rücken oder nicht? Würden wir es als Individualisierungsweisen verstehen, daß koreanische Eheleute der gebildeten Schichten im 16. Jahrhundert in jeweils eigenen Räumlichkeiten aßen und schliefen ${ }^{15}$ ?

Diese vielen Fragen lassen sich im Moment keineswegs beantworten. Ich will sie aber stellen, weil sich nur vor dem Hintergrund dieser komplexen Bezüge angemessene Antworten finden lassen. Was also bedeutet eine Selbstbeschreibung in den Abhängigkeiten von Patronagebeziehungen wirklich? Über welche Räume und Handlungsmöglichkeiten (ver-)handelten die Verfasser, wenn sie über Patronage schrieben? Welche Rolle spielten diese Selbstbeschreibungen im Kontext ihrer Gesellschaft?

In den letzten Jahrzehnten haben die Mediävistik und die Frühneuzeitforschung eine ganze Reihe von Erkenntnissen gewonnen, die für eine erneute Beschäftigung mit dem Thema grundlegend sind ${ }^{16}$. So hat Hans Medick darauf hingewiesen, daß Geschichtskonstruktionen wie die der Individualisierung eine zentristische Sichtweise implizieren; zu Recht kritisiert er, daß historische Prozesse dabei auf einen Aspekt reduziert und als bloße Vorgeschichte (der gegenwärtigen eigenen Verhältnisse) ihrer Eigenständigkeit und Fremdheit entkleidet werden. Dieter Kartschoke

13 Kritisch zum Stellenwert von Selbstzeugnissen zuletzt: Andrea Griesebner, Christina Lutter, Geschlecht und „Selbst“ in Quellen des Mittelalters und der Frühen Neuzeit, in: Vom Individuum zur Person. Neue Konzepte im Spannungsfeld von Autobiographietheorie und Selbstzeugnisforschung, hrsg. v. Gabriele Jancke, Claudia Ulbrich (Querelles. Jahrbuch für Frauen- und Geschlechterforschung 10, Göttingen 2005) 51-70, hier 59-62; Scheutz, Tersch, Individualisierungsprozesse; Marian Füssel, Die Rückkehr des ,Subjekts` in der Kulturgeschichte. Beobachtungen aus praxeologischer Perspektive, in: Historisierte Subjekte - Subjektivierte Historie. Zur Verfügbarkeit und Unverfügbarkeit von Geschichte, hrsg. v. Stefan Deines, Stephan Jaeger, Ansgar Nünning (Berlin, New York 2003) 141-159, v. a. $147 f .157$.

$14 \mathrm{~S}$. die vorsichtige und methodisch differenzierende Interpretation mittelalterlicher literarischer Phänomene von Kartschoke, Ich-Darstellung.

15 Ich beziehe mich damit auf den im Sommersemester 2004 an der FU Berlin gehaltenen Vortrag von Sungjong Paik, Mein lieber Mann, warum rühmst Du Dich so? Geschlechterbeziehungen im Korea des 16. Jahrhunderts (über den Briefwechsel eines Gelehrten mit seiner Ehefrau).

16 Vgl. Gabriele Jancke, Autobiographie als soziale Praxis. Beziehungskonzepte in Selbstzeugnissen des 15. und 16. Jahrhunderts im deutschsprachigen Raum (Selbstzeugnisse der Neuzeit 10, Köln, Weimar, Wien 2002), im folgenden zitiert: Jancke, Autobiographie als soziale Praxis; ferner dies., Rez. zu: Entdeckung des Ich. Die Geschichte der Individualisierung vom Mittelalter bis zur Gegenwart, hrsg. v. Richard van Dülmen (Köln, Weimar, Wien 2001), in: L'Homme Z.F. G. 13,2 (2002) 297-300. 
hat für volkssprachliche literarische Texte des Mittelalters festgestellt, daß einzelne Spuren von Individualität sehr wohl und zahlreich zu finden, aber jeweils auf nicht-individualistische Gesellschaftskonzepte bezogen und daraus erst recht keine zusammenhängenden Entwicklungslinien rekonstruierbar seien. Mit anderen Worten: Auch wenn man etwas feststellt, was dem eigenen Begriff von Individualität entspricht, heißt dies noch lange nicht, daß in den Quellen eine solche Art von Individualität zum Ausdruck gebracht wurde. Erst durch sorgfältige und zum Teil aufwendige Kontextualisierung könnte dies wirklich geklärt werden. Eva Schlotheuber hat gezeigt, daß im Mittelalter nicht die Individualität als Wert galt, sondern daß die subjektive Aneignung von allgemein gültigen Normen angestrebt wurde, so daß spätmittelalterliche und frühneuzeitliche Phänomene auch vor diesem Hintergrund nicht einfach als Vorgeschichte moderner Individualität verstanden werden können. Peter Burke hat auf die ethnozentrischen Engführungen aufmerksam gemacht, die Individualität und individualistische Selbstkonzepte ebenso wie autobiographisches Schreiben als spezifisch für die westliche europäische Kultur erklären, während ein kurzer Blick etwa auf Japan oder China schnell ein anderes Bild erkennen läßt. Kritisch hat auch August Nitschke notiert, daß in den Individualitätskonzepten der Geschichtswissenschaft ein eurozentrisches Personkonzept zum Maßstab der Kulturen aller Gegenden und Zeiten gemacht und daß genau dies zum Kern einer einzigen Natur des Menschen erklärt wird. Schließlich ergibt sich aus den Beiträgen von Caroline Walker Bynum, Natalie Zemon Davis und David Sabean eine grundlegende Infragestellung des Konzepts vom isolierten, autonomen Individuum, wie es in der Forschung meist verwendet wird: Die Frage nach dem Individuum ist falsch gestellt, wenn dabei klare und feste Grenzen vorausgesetzt sind, die eine für sich stehende Person umschreiben. Derlei Grenzen umfaßten für frühneuzeitliche Menschen vielmehr ganze soziale Gebilde mitsamt ihren internen sozialen Verflechtungen und Machtverhältnissen. Einzelne Menschen, Männer genau wie Frauen, handelten und verstanden sich von ihren Positionen her, die sie innerhalb dieser Netzwerke einnahmen. Verhältnisse und Beziehungen $\mathrm{zu}$ anderen waren von vornherein Bestandteil, zugleich aber Voraussetzung der Individualität einzelner Menschen ${ }^{17}$.

17 Hans Medick, „Missionare im Ruderboot“? Ethnologische Erkenntnisweisen als Herausforderung an die Sozialgeschichte, in: Alltagsgeschichte. Zur Rekonstruktion historischer Erfahrungen und Lebensweisen, hrsg. v. Alf Lüdtke (Frankfurt a. M., New York 1989) 48-84; Kartschoke, Ich-Darstellung; Eva Schlotheuber, Norm und Innerlichkeit. Zur problematischen Suche nach den Anfängen der Individualität, in: ZHF 31,3 (2004) 329-357; Peter Burke, Representations of the Self from Petrarch to Descartes, in: Rewriting the Self. Histories from the Renaissance to the Present, hrsg. v. Roy Porter (London, New York 1997) 1728; August Nitschke, Gegen eine anthropozentrische Geschichtswissenschaft. Die Bedeutung der nichteuropäischen Kulturen für den Standort der Historiker, in: HZ 265,2 (1997) 281-307; Caroline Walker Bynum, Did the Twelfth Century Discover the Individual?, in: Journal of Ecclesiastical History 31,1 (1980) 1-17, überarb. in: dies., Jesus as Mother. Studies in the Spirituality of the High Middle Ages (Berkeley, Los Angeles, London 1982) 82-109; Natalie Zemon Davis, Boundaries and the Sense of Self in Sixteenth-Century France, in: Reconstructing Individualism. Autonomy, Individuality, and the Self in Western Thought, 
Im folgenden werden die Patronageverhältnisse, wie sie in den Selbstzeugnissen der Gelehrten des 15. und 16. Jahrhunderts sichtbar werden, auf die neuzeitlichen Individualisierungsprozesse bezogen. Ich greife also die klassischen soziologischen Zugänge auf und gehe davon aus, daß Individualisierung nicht nur in sozialen Strukturen stattfindet, sondern auch mit konkreten sozialen Beziehungen verbunden und ohne sie gar nicht möglich ist. Meine Frage lautet, inwiefern für Gelehrte die Patronage eine solche Beziehungsform war und auch in ihren autobiographischen Schriften bewußt so dargestellt wurde und was dies für ihre Handlungsräume bedeutete. Der Zugang erfolgt also nicht aus der Makroperspektive über die Frage, ob wir Phänomene finden, die wir als Individualität und in einem weiteren Deutungsschritt als Elemente eines größeren Individualisierungsprozesses identifizieren. Vielmehr geht es in einer mikrohistorischen Perspektive darum, wie die betreffenden Sachverhalte in ihrem eigenen Kontext und von den Akteuren selbst bewertet wurden, welchen Stellenwert sie dort hatten, ob und inwieweit und für wen sie auf längere Sicht prägend zu werden vermochten.

Die Selbstzeugnisse der Gelehrten benutze ich als Quellen, ohne aber zugleich anzunehmen, daß es sich dabei um die einzigen oder auch nur besonders wichtige Quellen für Individualisierungsphänomene in dieser sozialen Gruppe oder gar in der Gesellschaft insgesamt handle. Im Rahmen eines akteurzentrierten Ansatzes, wie er in der historischen Anthropologie etwa von Natalie Zemon Davis, Claudia Ulbrich oder Hans Medick vertreten wird, konzentriere ich mich auf Merkmale wie Sichtbarkeit, Anerkennung, Einfluß und Macht, Verfügung über Ressourcen, die ich durch die Fragen nach Patronageverhältnissen durchweg in sozialen Beziehungen einbette. Meine Untersuchungen bleiben auf eine kleine, wenngleich einflußreiche und durch die von ihren Mitgliedern so zahlreich produzierten schriftlichen Dokumente für uns überdurchschnittlich sichtbare gesellschaftliche Gruppe beschränkt. Mit dem hier vorgestellten zeitlichen Ausschnitt des 15. und 16. Jahrhunderts können die langdauernden, sich über Jahrhunderte hinweg erstreckenden Prozesse selbstverständlich nicht nachgezeichnet werden. Aber die vorgestellten Phänomene sind im Rahmen sowohl von lange bestehenden Patronagestrukturen als auch von langfristig verlaufenden Individualisierungsprozessen zu sehen.

hrsg. v. Thomas C. Heller, Morton Sosna, and David E. Wellbery with Arnold I. Davidson, Ann Swidler, and Ian Watt (Stanford 1986) 53-63, 332-335 (dt. Übers.: Bindung und Freiheit. Die Grenzen des Selbst im Frankreich des 16. Jahrhunderts, in: Natalie Zemon Davis, Frauen und Gesellschaft am Beginn der Neuzeit. Studien über Familie, Religion und die Wandlungsfähigkeit des sozialen Körpers [Berlin 1986] 7-18, 133-135); David Sabean, Production of the Self during the Age of Confessionalism, in: Central European History 29 (1996) 1-18 (dt. Übers.: Selbsterkundung. Beichte und Abendmahl, in: Entdeckung des Ich. Die Geschichte der Individualisierung vom Mittelalter bis zur Gegenwart, hrsg. v. Richard van Dülmen [Köln, Weimar, Wien 2001] 145-162). 


\section{Patronage}

Patronage ist ein Thema, das sich bei den autobiographischen Texten des 15. und 16. Jahrhunderts geradezu von selbst aufdrängt. Von anderen Menschen, so viel fällt unmittelbar ins Auge, ist in den etwa 230 Schriften, die ich untersucht habe, auf Schritt und Tritt die Rede, so viel sogar, daß ein Teil der Autobiographieforschung sich Sorgen macht, ob es sich überhaupt um richtige autobiographische Texte handle oder ob nicht dafür allzuwenig von der eigenen Person geschrieben werde. Festzuhalten ist jedenfalls, daß es gerade die männlichen und insbesondere die gelehrten Verfasser waren, die sich selbst in sozialen Beziehungen darstellten und im Verhältnis zu anderen definierten - keineswegs als das autonome, isolierte, auf sich bezogene Individuum, das man bevorzugt in Autobiographien und insbesondere bei männlichen Autoren zu suchen pflegt.

Beziehungen waren für Männer, dies zeigen die autobiographischen Quellen sehr nachdrücklich, über die schon vielfach untersuchten Bereiche von Sexualität und Ehe hinaus auch in ganz anderen Handlungsfeldern von Bedeutung. Die Patronage ${ }^{18}$ soll hier als exemplarischer Beziehungstyp vorgestellt werden, der weder auf familiäre oder sexuelle Nahbereiche noch auf heterogeschlechtliche Konstellationen festgelegt ist, auch nicht von vornherein geschlechterexklusive Rollendefinitionen beinhaltet, und der schließlich persönliche mit öffentlichen Aspekten verbinden kann. Die Merkmale von Patronagebeziehungen sind schnell genannt: Ungleichheit, Ressourcentausch, persönlicher Charakter, langfristige Beziehung ohne rechtliche Regelungen, gegenseitige Verpflichtung ${ }^{19}$. Bleibt noch, sich die

18 Das Folgende beruht auf Jancke, Autobiographie als soziale Praxis, Kap. 2: Abhängig sein: Gelehrte Männer in Patronageverhältnissen. Rekonstruktion einer Gruppenkultur, 75-165, detaillierte Nachweise finden sich dort; vgl. weiterhin dies., Early Modern Scholars' Patronage Networks and Their Representation by Autobiographical Writers (16 ${ }^{\text {th }}$ Century), in: Kakanien revisited [20.4.2004], URL: http://www.kakanien.ac.at/beitr/ncs/GJancke1.pdf. - Einige grundlegende Titel: Wolfgang Reinhard, Freunde und Kreaturen. „Verflechtung“ als Konzept zur Erforschung historischer Führungsgruppen. Römische Oligarchie um 1600 (Schriften der Philosophischen Fachbereiche der Universität Augsburg 14, München 1979), im folgenden zitiert: Reinhard, Freunde und Kreaturen; Katarina Sieb-Burens, Oligarchie, Konfession und Politik im 16. Jahrhundert. Zur sozialen Verflechtung der Augsburger Bürgermeister und der Stadtpfleger 1518-1618 (Schriften der Philosophischen Fakultäten der Universität Augsburg 29, München 1986); Simon Teuscher, Bekannte - Klienten - Verwandte. Soziabilität und Politik in der Stadt Bern um 1500 (Norm und Struktur 9, Köln, Weimar, Wien 1998); Peter Burke, History and Social Theory (Cambridge 1992) 71-75: Patronage and Corruption; S. N. Eisenstadt, L. Roniger, Patrons, Clients and Friends. Interpersonal Relations and the Structure of Trust in Society (Themes in the Social Sciences, Cambridge etc. 1984); Jeremy Boissevain, Friends of Friends: Networks, Manipulators, and Coalitions (Oxford 1974).

19 Für diese Merkmale vgl. Richard P. Saller, Personal Patronage Under the Early Empire (Cambridge etc. 1982) 1 (nach Boissevain) sowie ch. 1: The language and ideology of patronage, 7-39, im folgenden zitiert: Saller, Personal Patronage, und Ronald Weissman, Taking Patronage Seriously: Mediterranean Values and Renaissance Society, in: Patronage, Art, and Society in Renaissance Italy, hrsg. v. F. W. Kent, Patricia Simons, J. C. Eade (Humanities Research Centre, Oxford University Press Series 1, Canberra, Oxford 1987) 25-45, hier 25f., 
persönlichen und gesellschaftlichen Dimensionen dieses Beziehungstyps näher anzusehen ${ }^{20}$.

Die wichtigsten, jedenfalls die intensivsten Erfahrungen mit anderen machen Menschen in verschiedenen Arten von persönlichen Beziehungen. Zu ihnen gehört die Patronage. Das Kennzeichen solcher Beziehungen ist, daß sie auf Vertrauen beruhen und gleichzeitig in einem - jedenfalls sehr weitgehend - rechtsfreien Raum angesiedelt sind. Sehr häufig handelt es sich dabei um Beziehungen zwischen Ungleichen, etwa im Hinblick auf Alter oder Geschlecht, und damit ist in der Regel die Ungleichheit jeweils verfügbarer Ressourcen wie Erfahrung, materielle Güter, physische Stärke und geistige Überlegenheit, Zugang zu sozialen Räumen und weiteren sozialen Beziehungen mitsamt den daran hängenden Handlungs- und Unterstützungsmöglichkeiten verbunden. Gesucht und angeboten - wenngleich oft nicht wirklich gegeben - werden Fürsorge, Versorgung, Schutz, Förderung und Unterstützung, Würdigung, Gesellschaft, Beratung, Liebe. Aufgrund dessen spielt es in persönlichen, auf Vertrauen beruhenden Beziehungen eine entscheidende Rolle, wie die Beteiligten mit materiellen, physischen oder emotionalen Bedürfnissen und Abhängigkeiten umgehen. Vor allem ist auf die ungleichen Machtverhältnisse zu achten, die dem stärkeren Part mannigfache Möglichkeiten zur Aus-

im folgenden zitiert: Weissman, Taking Patronage Seriously; John W. Bennett, Art. Paternalism, in: International Encyclopedia of the Social Sciences, hrsg. v. David L. Sills (New York 1968) vol. 11, 472-477, hier 475-477 (Patronage als eine bestimmte Spielart von Paternalismus). Was das paternalistische Grundmuster für Geschlechterverhältnisse bedeutet, ist in diesen Definitionen nicht reflektiert; vgl. aber Ina Schabert, Englische Literaturgeschichte. Eine neue Darstellung aus der Sicht der Geschlechterforschung (Stuttgart 1997) 98-121, im folgenden zitiert: Schabert, Englische Literaturgeschichte, die sich mit den ambivalenten Haltungen männlicher Literaten als Klienten gegenüber ihren adligen Patroninnen befaßt. In konkreten Forschungsarbeiten werden nicht immer, aber zumeist Männer als Agierende untersucht. Wie Patronage überhaupt, so ist auch die Rolle von Frauen in Patronagebeziehungen für den deutschsprachigen Bereich kaum erforscht; zur neueren Lit. s. aber Heiko Droste, Patronage in der Frühen Neuzeit - Institution und Kulturform, in: Zeitschrift für historische Forschung 30 (2003) 555-590, sowie Birgit Emich, Nicole Reinhardt, Hillard von Thiessen, Christian Wieland, Stand und Perspektiven der Patronageforschung. Zugleich eine Antwort auf Heiko Droste, in: ebd. 32 (2005) 233-265. Aus der Fülle von Arbeiten über Frauen als Patroninnen vgl. hier nur: Merry E. Wiesner, Women and Gender in Early Modern Europe (New Approaches to European History 1, Cambridge 1993) 140-143, 145; Charmarie Jenkins Blaisdell, Calvin's Letters to Women: The Courting of Ladies in High Places, in: Sixteenth Century Journal 13,3 (1982) 67-84; Silent But for the Word: Tudor Women as Patrons, Translators, and Writers of Religious Works, hrsg. v. Margaret Patterson Hannay (Kent 1985); Amy Katz Kaminsky, Elaine Dorough Johnson, To Restore Honor and Fortune: "The Autobiography of Leonor López de Córdoba", in: The Female Autograph. Theory and Practice of Autobiography from the Tenth to the Twentieth Century, hrsg. v. Domna C. Stanton (Chicago, London 1987) 70-80, hier 72; Sharon Kettering, The Patronage Power of Early Modern French Noblewomen, in: Historical Journal 32 (1989) 817-841; Carolyn Valone, Piety and Patronage: Women and the Early Jesuits, in: Creative Women in Medieval and Early Modern Italy. A Religious and Artistic Renaissance, hrsg. v. E. Ann Matter, John Coakley (Philadelphia 1994) 157-184.

20 Dies und das Folgende nach Jancke, Autobiographie als soziale Praxis 75-82. 
nutzung des schwächeren Teils und zum Mißbrauch in die Hände spielen, in aller Regel für andere kaum erkennbar und in jedem Fall schwer zu verhindern.

Bei Patronagebeziehungen werden im Unterschied zu Eltern-Kinder- oder EheBeziehungen weder Ungleichheiten von Alter oder Geschlecht noch die gesellschaftlich vorgeprägten, institutionalisierten Machtverhältnisse als konstitutiv angesehen. Patronagebeziehungen werden im Kontext eines paternalistischen Gesellschaftsverständnisses verortet und als ein persönliches, langfristiges Verhältnis zumeist unter Erwachsenen, vielfach solchen gleichen Geschlechts, verstanden. Ungleich sind die Ressourcen, über die die BeziehungspartnerInnen - Männer oder Frauen, wenngleich in den autobiographischen Schriften Frauen nur selten und dann nur als Patroninnen sichtbar werden - jeweils verfügen, sowohl in der Art als auch im Umfang. Die Beteiligten machen sich diese gegenseitig zugänglich und tauschen sie miteinander aus. Es ist daher Raum für sehr verschiedene Personenkonstellationen, für unterschiedlichste Verwendungsweisen der ausgetauschten Ressourcen und für Veränderungen der Beziehung - allerdings verfügen die Beteiligten über sehr unterschiedliche Handlungsspielräume.

In emotionaler Hinsicht kann die Patronagebeziehung in einem breiten Spektrum zwischen nüchterner, distanzierter Kalkulation des jeweiligen Nutzens und engem, umfassendem Freundschaftsverhältnis gestaltet werden. Darüber hinaus läßt sich für die römische Antike wie auch für die Frühe Neuzeit innerhalb von Patronagebeziehungen geradezu eine entsprechende Rhetorik feststellen: Wenn die Beteiligten sich gegenseitig als Freunde bezeichneten oder wenn grundsätzlicher von Freundschaft gesprochen wurde, dann war in vielen Fällen ein Patronageverhältnis gemeint ${ }^{21}$.

In sozialer Hinsicht kann ein Patronageverhältnis eine Vielfalt von Handlungsfeldern und Menschen verschiedener gesellschaftlicher Schichten und Räume verbinden, schließt aber zugleich auch andere Menschen aus. Zudem ist dieser Beziehungstyp nicht wie die Eltern-Kinder- und die Ehe-Beziehungen allein durch eine dyadische, bipolare Konstellation gekennzeichnet, sondern zugleich durch die mögliche Einbindung in weitgespannte Netzwerke, in denen die Patronagebeziehung für die Beteiligten den Zugang auch zu anderen Beziehungspartnern und den

21 Für die römische Antike vgl. Saller, Personal Patronage, bes. 11-15; für die Frühe Neuzeit Guy Fitch Lytle, Friendship and Patronage in Renaissance Europe, in: Patronage, Art, and Society in Renaissance Italy, hrsg. v. F. W. Kent, Patricia Simons, J. C. Eade (Humanities Research Centre, Oxford University Press Series 1, Canberra, Oxford 1987) 47-61, weiterhin Reinhard, Freunde und Kreaturen 37-39. Reinhard hält die Sprache der Freundschaft in Patronagebeziehungen für einen „Mythos“ (39) und nimmt an, daß beide Beziehungstypen sich tatsächlich klar gegeneinander abgrenzen lassen, daß es also keine Überschneidungen gäbe: „Wird das Ungleichgewicht unter Freunden allzu eindeutig oder gar permanent, dann ist die Grenze zum Phänomen der Patronage überschritten. “ (38). Es ist Reinhard zuzustimmen, wenn er Freundschaft und Patronage für jeweils eigene Phänomene hält, die nicht unbedingt deckungsgleich sein mußten. Zumindest für die Frühe Neuzeit ist m. E. aber davon auszugehen, daß es auch einen Bereich gab, in dem sich beide Beziehungstypen überschneiden konnten - d. h. es gab Beziehungen, die sowohl ein Freundschafts- als auch ein Patronageverhältnis darstellten, und andere, die entweder das eine oder das andere waren; vgl. dazu ähnlich Weissman, Taking Patronage Seriously $30 \mathrm{f}$. 
von ihnen kontrollierten Ressourcen eröffnen kann. Diejenigen, die an diesen Beziehungen und Netzwerken nicht beteiligt werden, müssen durch ihr Ausgeschlossensein persönliche Vorteile entbehren, können sich unter Umständen aber auch wirkungsvoll organisierten, systematischen Benachteiligungen oder gar Schädigungen ausgesetzt sehen. Eventuell organisieren sie sich in eigenen Netzwerken. Dieser Gesichtspunkt zeigt, daß Patronage immer auch in Gruppenkulturen eingebettet ist, die über die Aufnahme konkreter Personen in die Netzwerke oder ihren Ausschluß gewisse Vorentscheidungen treffen oder zumindest Präferenzen ausbilden.

In der Frühen Neuzeit durchzogen Patronageverhältnisse die Gesellschaft in ihren unterschiedlichen Teilen, Schichten, Milieus und Handlungsfeldern. Von der Patronageforschung sind meist nur ausgewählte Handlungsfelder - wie Kunst, Literatur, Politik - und ausgewählte Milieus - vor allem Höfe und die römisch-katholische Kirche - in den Blick genommen worden ${ }^{22}$. Dabei konzentrierten sich die Untersuchungen auf die jeweiligen Oberschichten, auf die von ihnen kontrollierten Führungspositionen und die von ihnen unmittelbar in Dienst genommenen intellektuellen, literarischen und künstlerischen Arbeitskräfte. Dabei hat sich gezeigt, $\mathrm{daß}$ in vielen gesellschaftlichen Teilbereichen Patronagebeziehungen zu den selbstverständlichen Formen des sozialen Umgangs gehörten und daß sie das Wissen darüber, auf welche Weise Menschen und Projekte voranzubringen waren, mit prägten.

22 Kunst: u. a. Patronage, Art, and Society in Renaissance Italy, hrsg. v. F. W. Kent, Patricia Simons, J. C. Eade (Humanities Research Centre, Oxford University Press Series 1, Canberra, Oxford 1987); Peter Burke, Die Renaissance in Italien. Sozialgeschichte einer Kultur zwischen Tradition und Erfindung (Berlin 1984, zuerst engl. 1972), v. a. Kap. Auftraggeber und Förderer 85-116; Bernd Roeck, Kunst-Patronage in der Frühen Neuzeit. Studien zu Kunstmarkt, Künstlern und ihren Auftraggebern in Italien und im Heiligen Römischen Reich (15.-17. Jahrhundert). Mit 33 Abb. (Sammlung Vandenhoeck, Göttingen 1999). Literatur: u. a. Suzanne R. Westfall, Patrons and Performance. Early Tudor Household Revels (Oxford 1990); Schabert, Englische Literaturgeschichte 98-121. Politik: u. a. Klientelsysteme im Europa der Frühen Neuzeit, hrsg. v. Antoni Maczak unter Mitarb. v. Elisabeth MüllerLuckner (Schriften des Historischen Kollegs. Kolloquien 9, München 1988); Patronage und Klientel. Ergebnisse einer polnisch-deutschen Konferenz, hrsg. v. Hans-Heinrich Nolte (Beihefte zum Archiv für Kulturgeschichte 29, Köln, Wien 1989); Gunner Lind, Great Friends and Small Friends: Clientelism and the Power Elite. In: Power Elites and State Building, hrsg. v. Wolfgang Reinhard (The Origins of the Modern State in Europe, $13^{\text {th }}$ to $18^{\text {th }}$ Centuries, Oxford 1996) 123-147. Vgl. ferner zu Politik, Kirche, Kunst, Literatur und Theater die Beiträge in: Patronage in the Renaissance, hrsg. v. Guy Fitch Lytle, Stephen Orgel (Folger Institute Essays, Princeton 1981). Höfe: u. a. Princes, Patronage and the Nobility. The Court at the Beginning of the Modern Age, c. 1450-1650, hrsg. v. Ronald G. Asch, Adolf M. Birke (Studies of the German Historical Institute, Oxford 1991); Sharon Kettering, Patrons, Brokers, and Clients in Seventeenth-Century France (New York, Oxford 1986); Linda Levy Peck, Court Patronage and Corruption in Early Stuart England (London 1993); ferner Westfall, Patrons and Performance. Kirche: u. a. Wolfgang Reinhard, Nepotismus. Der Funktionswandel einer papstgeschichtlichen Konstante. In: Zeitschrift für Kirchengeschichte 86 (1975) 145-185; ders., Kirche als Mobilitätskanal in der frühneuzeitlichen Gesellschaft, in: ders., Ausgewählte Abhandlungen (Historische Forschungen 60, Berlin 1997) 5373 (zuerst 1988); Nicole Reinhardt, Macht und Ohnmacht der Verflechtung. Rom und Bologna unter Paul V. Studien zur frühneuzeitlichen Kirchenpolitik im Kirchenstaat (Frühneuzeit-Forschungen 8, Tübingen 2000). 
In anderen Studien wurden Patronageverhältnisse auch in städtischen Milieus nachgewiesen. Darüber hinaus wurde hier vor allem deutlich, wie weit und verzweigt Patronage alle Schichten der Gesellschaft durchzog. Nicht zuletzt konnten alle Arten von Dienstverhältnissen zu Patronagebeziehungen ausgebaut werden.

In einem weiterführenden Aufsatz hat Ronald Weissman vor einigen Jahren gezeigt, daß Patronageverhältnisse zumindest in der italienischen Renaissancegesellschaft des 14. und 15. Jahrhunderts über Teilgebiete weit hinausreichten und insgesamt als grundlegende gesellschaftliche Struktur einzuschätzen sind ${ }^{23}$. Patronage war demnach nicht etwa ein feudaler Überrest und damit eine Art der Strukturierung ländlicher Gesellschaften, sondern eine Form städtischer Organisation und als mehrfachrelationale Beziehung stets in verzweigte und weit ausgreifende Netzwerke eingebettet. Weissman warnt davor, Patronage nur als hierarchische Bindung von sozial Ungleichen anzusehen, und betont statt dessen die Einbeziehung von auch sozial Gleichrangigen.

Bei diesem System von Werten und Verhaltensweisen handelte es sich um ein informelles soziales Ethos, so daß die danach gestalteten Beziehungen eher auf einer moralischen denn einer rechtlichen Grundlage beruhten. Der zentrale Punkt dieses Ethos bestand darin, daß die Welt in Freunde und Fremde geteilt wurde. In dieser Welt kam es darauf an, Freunde zu fördern, Dankbarkeit gegenüber Patronen zum Ausdruck zu bringen und Loyalität gegenüber Verwandten zu zeigen. Gegenüber Fremden hingegen waren Verhaltensweisen einer Konkurrenzsituation angebracht, in der man nicht alle unparteilich und neutral zu behandeln suchte. Patronage war - und ist - Teil einer Welt, in der Gaben und Verpflichtungen die gegenseitigen Beziehungen bestimmen. Was Patronage leistet, ist: Sie ist einer der Beziehungstypen, mit denen Menschen in einer solchen Welt Distanzen und Unterschiede wahren und gleichzeitig gemeinsame soziale Räume und Zugehörigkeiten herstellen können. Die Gestaltung dieses Beziehungstyps setzt Ungleichheit voraus und sucht darauf durch die Schaffung einer Beziehung einen sozialen Reim zu machen - mit allen Möglichkeiten für den mächtigen Teil, von der schamlosen Ausnutzung der Abhängigkeit bis hin zur selbstlosen Förderung anderer.

\section{Gelehrte Männer in Patronageverhältnissen}

Was an diesen Patronagebeziehungen für die gelehrten Autoren von Interesse war, waren in aller Regel sehr greifbare Dinge. Es ging ihnen um Ressourcen, die ihnen nur ein Patron und die persönliche Beziehung zu ihm zugänglich zu machen vermochten. Es konnte sich dabei um materielle Unterstützung für ein Studium (durch ein Stipendium) oder für gelehrte Projekte und Bücher handeln - wie etwa bei Sebastian Leonhart (1544-1610) oder Jakob Andreae (1528-1590) -, es konnte um Stellen und ihre Vermittlung gehen oder aber darum, zum Unterricht bei einem bestimmten Lehrer aufgenommen zu werden oder Kontakte vermittelt zu bekom-

23 Weissman, Taking Patronage Seriously. 
men, zu anderen Gelehrten und Lehrern, zu wohlhabenden oder allgemein förderungswilligen Patronen - wie bei Daniel Greiser (1504-1591), Lukas Geizkofler (1550-1620) oder Simon Lemnius (1511-1550). Als Patrone kamen vor allem Lehrer und Professoren, potente Geld- und potentielle Arbeitgeber (wie Adlige, Firmenchefs, hohe Geistliche, Landesherren, Stadträte) oder auch Verwandte in Frage ${ }^{24}$.

Es zeigt sich in diesem Zusammenhang, daß soziale Beziehungen eine Ressource darstellten, die die Handlungsmöglichkeiten der Beteiligten erheblich erweitern konnten. Ein weiterer interessanter Punkt betrifft die gelehrten Qualifikationen der Verfasser: Keineswegs bedeutet Patronage, daß die gelehrten Klienten etwa nur durch ihre Beziehungen und ohne Qualifikationen an die begehrten Ressourcen herankamen. Ganz im Gegenteil, Patronage war zumindest in diesem Milieu nicht ohne Qualifikation zu haben, und sie sollte ihrerseits vielfach den Erwerb von Qualifikationen ermöglichen helfen ${ }^{25}$. Damit war die Qualifikation des Klienten auch eine Ressource für die Beziehung selbst und für den Patron, konnte sie doch dem Klienten Zugang zu Patronen verschaffen und umgekehrt das Ansehen des Patrons durch einen erfolgreichen und tüchtigen Klienten steigern.

Nicht immer mußte Patronage eine große soziale Differenz und eine Hierarchie zwischen Patron und Klient beinhalten. Patronagebeziehungen konnten auch Gelehrte untereinander knüpfen - wie es sich etwa bei Johannes Trithemius (14621516) oder Hieronymus Wolf (1516-1580) beobachten läßt -, und die Differenzen und Hierarchien konnten sich im Laufe der Zeit verschieben, ja sogar umkehren, so daß der erfolgreiche ehemalige Schüler - z. B. Melchior Khlesel (1552-1630) etwa für seinen ehemaligen Lehrer und Patron nun selbst als Patron tätig wurde. Patronage konnte die Möglichkeit bedeuten, zwischen verschiedenen Patronen zu wählen und sich somit als Klient Handlungsspielräume zu erschließen, wie bei Lukas Geizkofler; sie konnte aber auch zu fast ausweglosen, existentiellen Abhängigkeiten führen, die höchstens durch radikale Schritte zu beenden waren, wie bei Stephan Isaac (1542-1597).

Fast immer aber stellte Patronage eine Form der Beziehung dar, die als gegenseitige Würdigung praktiziert wurde und dafür verschiedene, ritualisierte Handlungsformen vorsah: die mündliche oder schriftliche Empfehlung eines Klienten, die Widmung einer gedruckten Schrift an einen oder auch mehrere Patrone, die Ehrung eines Patrons ${ }^{26}$. Die Nennung Friedrichs des Weisen durch Martin Luther (1483-1546) etwa ist als eine solche Ehrung zu verstehen, die den Patron in seiner Rolle anerkennen und hervorheben sollte. Damit wird der autobiographische Text selbst zu einer Form des Patronagehandelns, Teil einer praktizierten Patronagebeziehung. Vielfach ist das auch bei anderen autobiographischen Schriften der Fall, vor allem als Empfehlungsschreiben - etwa bei Josias Marcus (1524-1599) - oder Widmungsvorreden - etwa bei Gerhard Oemeken (1485-1562) oder Bartholomäus Scultetus (1540-1614).

${ }^{24}$ Jancke, Autobiographie als soziale Praxis 103-134 (Ressourcen), 93-99 (Patrone).

25 Ebd. 84, 111f., 120-122, 125, 139-141, 162.

26 Ebd. 135-161. 
Alle diese Handlungsformen zeigen, daß Patronage keine auf bloß zwei Beteiligte beschränkte Beziehung war. Dritte spielten hier stets eine unverzichtbare Rolle, so daß Patronagebeziehungen ansatzweise in ihrem Netzwerkcharakter erkennbar werden, angesiedelt in oft ausgedehnten und weit verzweigten Beziehungsnetzen, die auch nicht auf ein bestimmtes soziales Milieu beschränkt sein mußten. Worauf sie aber zumindest in den autobiographischen Darstellungen der christlichen Gelehrten begrenzt waren, das ist ein Gegenüber von Männern, auch wenn die faktische Präsenz von Frauen in solchen Netzwerken durchaus hie und $\mathrm{da}$ durchschimmert ${ }^{27}$. Patronage als eine Kultur der gegenseitigen Solidarität, Würdigung und Zuschreibung von Autorität unter Männern - das ist zwar keineswegs die vollständige soziale Wirklichkeit, aber doch genau das, was männliche Gelehrte schriftlich festhielten und als soziale Praxis mit ihren autobiographischen Schriften realisierten.

Man kann autobiographische Schriften als einzelne Texte für einzelne Personen und ihre Patronagebeziehungen lesen, aber auch als Quellen für konkrete Netzwerke und Typen von Netzwerken, die einem sagen, wie einzelne Gruppenkulturen in sich organisiert waren und wie unterschiedliche Gruppenkulturen miteinander verknüpft waren. Dabei ist es hilfreich, mit mehreren oder vielen Texten zu arbeiten, um möglichst viele und vielfältige Aspekte dieser Netzwerke in Erfahrung bringen zu können. In meinem Falle war es so, daß ich mit den zahlreichen autobiographischen Schriften gelehrter Verfasser, die sich als Quellen über Patronage lesen lassen, das Gelehrtenmilieu als eine Gruppenkultur mit ganz bestimmten Regeln und Ritualen rekonstruiert habe. Die wichtigsten Ziele darin waren, diesen Quellen zufolge, die gegenseitige Würdigung, die Selbstbeschreibung in Abhängigkeitsbeziehungen und in abhängigen Positionen sowie die Zuschreibung von Autorität an die Mitglieder dieser Netzwerke. Nicht zuletzt zeigt sich auch, daß das autobiographische Schreiben selbst ein Teil dieser gelehrten Gruppenkultur war.

In den autobiographischen Schriften von gelehrten Männern bildet dieser Beziehungstyp ein zentrales Element, von dem ihre Verhaltensweisen als eine eigene soziale Gruppe geprägt waren. Zusätzlich funktionierten die Texte dieser Gelehrten auch selbst im Rahmen von Patronagebeziehungen, d. h. sie sind ihrerseits als Teil von Patronagehandeln und als Teil solcher gruppenspezifischer sozialer Praktiken zu verstehen. Das will ich jetzt etwas näher ausführen.

Was zunächst auffällt, ist die Selbstverständlichkeit, mit der Patronagebeziehungen in diesen autobiographischen Texten präsent sind. Sie werden in aller Regel

27 Die Forschung schenkte bisher Frauen in Patronageverhältnissen ihre Aufmerksamkeit v. a. in der Rolle von Patroninnen. Zu Frauen als Klientinnen vgl. Mary Ellen Lamb, Patronage and Class in Aemilia Lanyer's Salve Deus Rex Judaeorum, in: Women, Writing, and the Reproduction of Culture in Tudor and Stuart Britain, hrsg. v. Mary E. Burke, Jane Donawerth, Linda L. Dove, Karen Nelson (Syracuse, New York 2000) 38-57; Jane Milling, "In the Female Coasts of Fame": Women's Dramatic Writing on the Public Stage 1669-71, in: Women's Writing [Great Britain] 7,2 (2000) 267-293. Beide Hinweise verdanke ich Sarah Heller Mendelson. 
nicht explizit benannt und erst recht nicht erklärt. Wenn Personen erwähnt und Handlungen berichtet werden, muß den Leserinnen und Lesern von selbst klar sein, in welchen Rollen die jeweiligen Personen dabei agieren, welchen Sinn diese Handlungen haben und nach welcher sozialen Logik sie zu verstehen sind. Das einschlägige Wissen über die allgemeinen Regeln, nach denen dieser Beziehungstyp funktioniert, wird ebenso vorausgesetzt wie ganz konkrete Kenntnisse über einzelne Personen und Beziehungen: Daß, um ein Beispiel zu nennen, Nicolaus Cusanus (1401-1464) seinen langjährigen Patron meint, wenn er in seiner Autobiographie Papst Eugen IV. nennt, ist nur dann verständlich, wenn man über den Patronagecharakter dieser Beziehung schon vorher Bescheid weiß. Diese Art der Darstellung sagt sehr viel darüber aus, welch lebendiges, vertrautes und legitimes Element Patronageverhältnisse in sozialen Wissensbeständen und Verhaltensrepertoires waren, zumindest in diesem sozialen Milieu.

Aber auch das Selbstverständnis christlicher Gelehrter wird dadurch beleuchtet. Bemerkenswert ist es, wie sie sich selbst in diesen Patronageverhältnissen verorteten: Sie zeichneten sich zumeist in der abhängigen Rolle des Klienten ${ }^{28}$. Was in ihren Texten hingegen kaum zur Sprache kommt, sind diejenigen Lebensbereiche, in denen sie selbst über Autorität und Einfluß gegenüber anderen verfügen konnten. Diejenigen sozialen Sensibilitäten, die sie von sich selbst autobiographisch formulierten, waren in Beziehungen zu Mächtigeren und zu den Gelehrtenkollegen angesiedelt; ihnen galten die autobiographische Aufmerksamkeit und Würdigung.

Auch wenn sich die Verfasser in Patronagebeziehungen selbst ganz überwiegend in der Klientenrolle zeigten, bedeutete dies keineswegs eine besondere Bescheidenheit oder gar Selbstabwertung, konnten sie doch gerade auf diese Art deutlich machen, daß sie des Vertrauens und der Wertschätzung eines einflußreichen und angesehenen Patrons würdig waren und diesem also wertvolle Ressourcen anzubieten hatten. Sowohl die eigene Person als auch die eigenen, in die Patronagebeziehung eingebrachten Ressourcen - z. B. in vielen Fällen akademische Bildung oder das Potential, solche zu erwerben und später etwa im Dienst des Patrons kompetent anzuwenden - konnten durch diese Form der Selbstdarstellung mit hohem sozialem Wert versehen werden.

Die Vorstellungen, die die gelehrten Autoren von Autorität entwickelt hatten, ließen neben ihrer eigenen keine andere Form von Autorität gelten. Andere Menschen, die weder über die gelehrte Autorität, die die Verfasser als Klienten demonstrierten, noch über soziale oder politische Autorität verfügten, die sie an ihren $\mathrm{Pa}$ tronen würdigten, kamen in diesem Selbst- und Beziehungskonzept gar nicht erst vor: Die Rituale gegenseitiger Wertschätzung, die die Autoren autobiographischer Schriften in Patronagebeziehungen zum Ausdruck brachten, räumten für solche andere keinen Platz ein. In diesen Schriften zeigt sich ein Verständnis von Individualität, das die Würdigung sozial einflußreicherer Patrone und umgekehrt die Autorisierung der eigenen Person der Verfasser durch diese Männer in den

28 Jancke, Autobiographie als soziale Praxis 91-102. 
Vordergrund rückte. Diese Individualität war von vornherein Teil einer Gruppenkultur, zutiefst von ihr geprägt, in ihr entstanden, auf sie hin orientiert. Ihre autobiographisch vorgetragenen Selbst- und Beziehungskonzepte entwickelten die Verfasser nach den Regeln dieser Gruppenkultur. Wie es vor allem an den Patronagebeziehungen greifbar wird, war Individualität gerade für die gelehrten Autoren grundsätzlich eine relationale Größe.

\section{Individualisierung als Ressource in Beziehungen}

Zusammenfassend läßt sich sagen: Wenn wir die autobiographischen Schriften von Gelehrten des 15. und 16. Jahrhunderts betrachten, erschließen sich gerade die in sozialen Beziehungen liegenden Individualisierungsweisen. Verschiedene Formen von Individualisierung haben sich gezeigt: Einerseits öffneten Patronagebeziehungen Zugänge zu Positionen, Handlungsmöglichkeiten und Ansehen für die je einzelne Person des Verfassers. Andererseits erhöhte das autobiographische Schreiben die Sichtbarkeit des Verfassers für andere, und das Schreiben über Patronagebeziehungen dürfte weiterhin Ansehen und Stellenwert des Autors befördert haben.

Angesichts der durch und durch hierarchisch strukturierten frühneuzeitlichen Gesellschaft sollte es vielleicht gar nicht so sehr verwundern, daß auch Gelehrte ihre Handlungsräume durch ihr Agieren in Abhängigkeitsbeziehungen wie der Patronage gestalten und erweitern konnten. Ein Patronageverhältnis mit seiner einerseits dyadischen Struktur und andererseits seiner Einbindung in größere soziale Netzwerke stellte für die Beteiligten flexible Möglichkeiten bereit, ihre Handlungsräume als einzelne Personen zu erweitern, und bot darüber hinaus auch gerade für die Gelehrten viele Gelegenheiten, sich und ihr Gegenüber öffentlich sichtbar zu machen, Ehre und Ansehen zu erhöhen ${ }^{29}$.

In ihren autobiographischen Schriften führen die Autoren uns sehr deutlich vor Augen, daß dies mit der ungleichen Verteilung von Ressourcen und Machtverhältnissen einherging, nicht aber aus solchen Ungleichheiten hinausführte. Die von ihnen gesuchten und demonstrierten Individualisierungsweisen mußten und konnten mit einem hierarchischen Gesellschaftsverständnis und der eigenen Position in einer hierarchischen Gesellschaft vereinbart werden. Die Gelehrten waren nach oben und nach unten in hierarchische Beziehungen eingebunden und fanden gerade darin ihre Möglichkeiten, sich persönlich zu profilieren und ihre Einflußmöglichkeiten als Einzelne an ihren jeweiligen Positionen zu gestalten. Ihr autobiographisches Schreiben als eine ihrer verschiedenen Individualisierungsweisen legt von diesen Komplexitäten beredtes Zeugnis ab. Nicht zuletzt zeigt es, daß Bindungen als eine Ressource für Individualisierung wirksam sein konnten - zugleich aber auch, daß sie es in sehr ungleicher Weise waren.

${ }_{29} \mathrm{Zu}$ den keineswegs bloß ,privaten“ AdressatInnenkreisen der autobiographischen Schriften s. Jancke, Autobiographie als soziale Praxis, Kap. 3: Für andere schreiben: Sprachen, Publikum, Publikationswege. Gruppenkulturen im Gesamtkorpus 166-210. 
Durch das autobiographische Schreiben wurden die anderen, die an diesen Bindungen beteiligt waren, von den Verfassern zudem in ungleicher Weise sichtbar bzw. unsichtbar gemacht: Während die über ihnen stehenden Patrone in den Texten den Fokus der Aufmerksamkeit von Publikum wie Verfasser bildeten und wie die Autoren selbst als Einzelpersonen benannt und gestaltet wurden, blieben die unter ihnen Stehenden meist unsichtbar. Diese Formen der Individualisierung waren nicht nur sozial in hohem Maße von konkreten und hierarchischen sozialen Orientierungen geprägt, sondern sie waren dies außerdem auch als Formen der schriftlichen (Selbst-)Darstellung.

Das autobiographische Schreiben selbst stellte also eine weitere, unmittelbar praktizierte Individualisierungsweise dar. Sie war für die Verfasser zwar ein Wert, aber nicht ihr Ziel. Unter anderem indem sie ihre didaktischen Absichten betonten oder andere Zwecke ihrer Texte benannten, machten sie vielmehr deutlich, daß es sich für sie um ein Mittel handelte, das erneut für die Gestaltung und Fortführung von Bindungen nutzbar sein sollte. Dabei nahmen sie dann selbst eine initiierende und prominente Rolle ein, denn sie waren es, die sich selbst und andere in ihren autobiographischen Schriften in ausgewählten Teilen sichtbar machten.

Was an diesem Testfall der Patronagebeziehungen deutlich wird, ist, daß Individualisierungsweisen nicht nur aus der Außenperspektive wahrnehmbar sind, sondern von den Verfassern dieser Texte im Rahmen der vorgefundenen sozialen Strukturen und mit Hilfe ihrer eigenen, gruppenspezifischen Ressourcen nachdrücklich gesucht und realisiert worden sind. Sofern im Vorangegangenen Individualisierungsweisen - auch mit längerfristigen Wirkungen - sichtbar geworden sind, waren sie entscheidend mit Macht über Menschen und Verfügungsgewalt über Ressourcen verknüpft. Könnte es sein, daß auch umgekehrt die Debatten um Individualität und Individualisierung unter anderem ein in verdeckter Form geführter Machtdiskurs sind?

Aus mikrohistorischer Perspektive rücken Individuum und Gesellschaft nahe zusammen und sind auch als analytische Begriffe nur tauglich, wenn sie in dieser Verflechtung und als mehrfachrelationale Kategorien verstanden werden. Aus der Perspektive der Akteure hatten einzelne Menschen, so hat sich in der Analyse der autobiographischen Darstellung von Patronageverhältnissen gezeigt, wohl ihren besonderen Wert, aber nicht als Individuen im Sinne von generalisierten, abstrakten, gleichwertigen Einzelnen. Vielmehr war der hier sichtbare individuelle Stellenwert geprägt von Stand, Geschlecht, Alter, Religion, wurde von den Verfassern also als Ausdruck der sozialen Position aufgefaßt. Als Kriterium für Individualisierung besaß die soziale Positionierung der jeweiligen AkteurInnen offenbar einen hohen Stellenwert. Besonders wichtig in diesem Zusammenhang waren die Faktoren von Macht und Abhängigkeit. Durch die Analyse von Patronageverhältnissen, wie sie in autobiographischen Texten dargestellt wurden, bringt der akteurzentrierte Ansatz die langfristigen Prozesse in ihrer Mikrostruktur und in größerer Komplexität zum Vorschein, als bisher bekannt war, und eröffnet so einen neuen Zugang zu einer Sozialgeschichte der Individualisierung, bei dem Beziehungen, Machtverhältnisse und Abhängigkeiten eine entscheidende Rolle spielen. Dieser 
Befund für eine europäische Gesellschaft öffnet weiterhin auch die Möglichkeit, für nicht-europäische Gesellschaften und ihre Patronagestrukturen neue Fragen zu stellen, die eventuelle Individualisierungsweisen mit einbeziehen.

$\mathrm{Ob}$ aber aus diesen Momenten auch schon zusammenhängende, langfristige Prozesse abgeleitet werden können oder gar ein bewußtes Hinwirken der Akteure auf die Entstehung solcher Prozesse, läßt sich nicht so einfach bejahen. Dafür ist das hier gezeichnete Bild viel zu fragmentarisch - so einflußreich die Gelehrten auch waren, und so wichtig für sie die Patronagebeziehungen als Individualisierungsweisen auch gewesen sein mögen, so wenig läßt sich doch auch nur ihre eigene soziale Gruppe aus den autobiographischen Texten allein beurteilen. So lange etwa gar nicht klar ist, ob möglicherweise bevorzugt die in Patronagebeziehungen erfolgreichen Gelehrten autobiographisch aktiv wurden, und so lange die autobiographischen Quellen nicht durch andere Quellen gegengelesen worden sind, und solange nicht neben Patronagebeziehungen auch andere Felder ins Auge gefaßt worden sind, ist noch nicht einmal für die Gruppe der Gelehrten ein abschließendes Urteil möglich. 
